\title{
Aplicação da modelagem matemática na operação de Estações de Tratamento de Esgotos por Lodos Ativados - o caso de Bragança Paulista
}

\author{
The use of mathematical modeling in the management of \\ Wastewater Treatment Plants operating under the activated \\ sludge process. The case study of Bragança Paulista's WWTP \\ Data de entrada: \\ $13 / 06 / 2017$ \\ Data de aprovação: \\ $31 / 10 / 2017$
}

\section{Resumo}

Modelos matemáticos podem e são utilizados para prever respostas de sistemas biológicos sujeitos a perturbações diversas, assim como alterações significativas das condições de afluência ou deterioração da qualidade do efluente. Por outro lado, a modelagem e a simulação permitem a adoção de estratégias de controle, com o objetivo de otimizar e adequar o funcionamento de uma ETE em uma dada condição, procurando-se garantir um melhor desempenho dos processos biológicos. Depois de apresentar as equações que regem o fenômeno, a metodologia baseada estritamente na DQQO (Demanda Química de Oxigênio) será aplicada a uma ETE (Estação de Tratamento de Esgoto) por lodos ativados, simulando-se alguns cenários e discutindo-se os resultados. Recorreu-se à ETE Bragança Paulista. Palavras-chave: Modelos Matemáticos. Demanda Química de Oxigênio. Taxa de Consumo de Oxigênio.

\section{Abstract}

Mathematical modeling can be used to predict responses to biological systems subjected to various disturbances, as well as significant changes in the conditions of affluence or deterioration of the quality of the effluent. On the other hand, the simulation and modeling allow for the adoption of control strategies in order to optimize and adapt the functioning of a Wastewater Treatment Plant (WWTP) in a given condition, to ensure a better performance of the biological processes. After presenting the equations governing the phenomenon, the methodology based strictly on COD (chemical oxygen demand) will be applied to a WWTP for activated sludge, simulating a few scenarios and discussing the results. The WWTP chosen was Bragança Paulista.

Keywords: Mathematical models. Chemical oxygen demand. Oxygen consumption rate 


\section{INTRODUÇÃO}

\subsection{Um pouco de história}

Giordano (2010) afirma que, ao longo dos últimos 30 anos, diversos modelos cinéticos foram criados para tentar descrever o comportamento do processo de degradação pelos microrganismos do lodo ativado. Em 1920, pela primeira vez foi aventada a remoção da matéria orgânica pelos microrganismos. A teoria da adsorção foi dominante nas décadas seguintes, sendo superada apenas em 1942 pela teoria da adsorção metabólica, segundo a qual, além da adsorção, ocorreria também o metabolismo microbiano. Entretanto, essa teoria não teve consenso científico, até que em 1955 pesquisadores começaram a considerar a composição do esgoto e suas relações nas reações físico-químicas e biológicas.

Em 1982, a International Association on Water Pollution Research and Control (IAWPRC) formou um grupo para analisar a modelagem matemática para projeto e operação em Processos de Lodo Ativados. Esse grupo baseou-se no que existia de mais avançado na época, liderado pelo professor Dr. G.v.R. Marais, da Universidade do Cabo, na África do Sul.

Em 1983, a Associação Internacional de Qualidade da Água (IAWQ ) formou um grupo de trabalho com o objetivo de promover a aplicação de modelos práticos para projeto e operação dos sistemas biológicos no tratamento de efluentes. 0 resultado final foi apresentado em 1987, e o modelo apresentado ficou sendo conhecido como Activated Sludge Model $\mathrm{n}^{\circ} 1$, ou simplesmente ASM1.

Alem Sobrinho (2012) faz um breve histórico de como a tecnologia se difundiu pelo mundo a partir da década de 1960, com as primeiras ETEs construídas para a remoção de nitrogênio, até o presente, passando pelas mais diversas opções, tais como sistemas mistos (com UASB), Bardenpho Modificado, etc.
Segundo Bye et al. (2012), "A modelagem matemática dos processos de Lodos Ativados de uma Estação de Tratamento de Esgotos é uma ferramenta poderosa que pode ajudar os tomadores de decisão a maximizar a capacidade operacional existente dessa planta, de modo a se obter o máximo benefício em novas ou futuras ampliações. Isso, sem dúvida, ajuda no desenvolvimento de programas de investimentos de capital de forma a acomodar o crescimento futuro".

\subsection{0 balanço de Massa}

Independentemente do tipo de processos e equipamentos, qualquer experimento estará sujeito, por mais complexo que seja tal experimento, às mesmas leis da física e da química, principalmente às que concernem à conservação de massa, energia e momentum.

De maneira simplificada, a lei da conservação de massa afirma que massa não pode ser criada nem destruída. Segundo Fernandes et al. (2006), podem ocorrer reações químicas, que transformam reagentes em produtos, mas a massa total (reagentes e produtos) não será alterada. Matematicamente pode-se escrever que:

$\frac{\mathrm{dm}}{\mathrm{dt}}=0 \quad$ ou $\quad\left(\begin{array}{l}\text { acúmulo } \\ \text { de } \\ \text { massa }\end{array}\right)=\left(\begin{array}{l}\text { massa } \\ \text { que } \\ \text { entra }\end{array}\right)-\left(\begin{array}{l}\text { massa } \\ \text { que } \\ \text { sai }\end{array}\right)$

Helou (2000) define modelo matemático como sendo uma simplificação de um sistema ou fragmento da natureza, realizado por meio de equações, sendo que a proximidade entre a representação e o sistema está diretamente ligada à precisão dos resultados. Assim, o usuário de um modelo pode pôr à prova diversas hipóteses, simulando situações existentes ou novas condições de operação. Um modelo pode ser usado para projetar um novo sistema ou até mesmo para prever um futuro comportamento. 
Segundo Vicente (2006), a simulação avalia as respostas de um sistema submetido a eventos em condições e restrições em uma operação em tempo real; já a simulação difere da otimização por não apresentar uma natureza otimizante, mas sim descritiva. A procura de uma solução ótima não é objetivo direto da simulação, uma vez que ela é extraída a partir de um grupo de possíveis resultados e variáveis de decisão.

O objetivo de uma simulação é basicamente conhecer mais profundamente as condições operacionais de uma planta existente, e dessa forma oferecer opções de melhorias ao processo. As simulações podem ser caracterizadas por duas formas distintas, a saber: a simulação física e a matemática.

A simulação física envolve o uso do próprio processo ou uma versão reduzida dele (planta piloto), sendo muitas vezes bastante dispendiosa e impossível de aplicar.

Já a simulação matemática se faz por meio das equações matemáticas dos principais fenômenos que envolvem o processo, mas pressupõe o conhecimento prévio de alguns coeficientes das equações envolvidas no processo. Seu desenvolvimento abrange os princípios básicos da conservação da massa e de energia. Sempre é importante a adoção de hipóteses simplificadoras, devendo-se sempre levar em consideração as condições iniciais e de contorno do problema em si.

Simulações de cenários em Sistemas de Lodos Ativados são de fundamental importância nas diversas etapas no planejamento de uma Estação de Tratamento de Esgoto (ETE), de duas formas distintas. Na fase de projeto, quando ainda é possível fazer mudanças no desenho da planta, e na fase de operação, quando já não se podem alterar dimensões de tanques, mas ainda é possível estabelecerem-se regras operacionais que minimizem os custos de operação da planta.
Para que uma Estação de Tratamento de Esgoto funcione de maneira adequada é necessário que as hipóteses adotadas na fase de planejamento sejam confirmadas na fase operacional. Para tanto é necessário que se estabeleçam cenários que minimizem custos operacionais, no caso de uma ETE já existente, ou que se diminuam custos de investimentos em ETEs a serem projetadas.

No caso de uma planta cujo tratamento seja feito por lodos ativados, os custos que têm o maior peso na composição do custo global são o de energia elétrica e o de transporte de lodo. Se, por um lado, o custo da energia elétrica varia de acordo com a região e o mercado energético, o de transporte dos lodos depende da distância entre a ETE e o aterro, um fator muito importante no caso de grandes metrópoles. Para tanto, é importante que se conheçam bem os principais parâmetros envolvidos no processo.

Para que se obtenham resultados compatíveis com a realidade operacional é necessário que, primeiramente, os dados sejam exaustivamente testados por meio de simulações matemáticas.

\section{OBJETIVO}

O objetivo deste trabalho foi o de estabelecer, por meio de um Balanço de Massa, baseado exclusivamente Demanda Química de Oxigênio (DQO), que é um parâmetro fácil de ser obtido, a quantidade mínima de estruturas a serem colocadas em operação (tanques de aeração e decantadores secundários), bem como estimar a energia elétrica a ser dispendida no tratamento, por meio do monitoramento da Taxa de Consumo de Oxigênio (TCO), mantendo-se restringidas a Taxa de Aplicação de Sólidos (TAS) nos decantadores secundários e a concentração de sólidos voláteis nos reatores $(\mathrm{XV})$. 
As análises de DBO, ou Demanda Bioquímica de Oxigênio, comumente utilizadas em trabalhos de engenharia, demoram cinco dias para que se obtenham resultados, enquanto as de DQO não demoram mais do que duas horas, além de existirem equipamentos que podem realizar a aquisição de dados em campo. Outro fator relevante é o seu custo, que é pelo menos quatro vezes maior. Em que se pese isso, a DBO tem a enorme desvantagem de não "fechar" um balanço de massa devido ao resíduo endógeno. ${ }^{1}$

\section{MATERIAIS E MÉTODOS}

É importante salientar que a hipótese fundamental para efeito dos cálculos pressupõe o regime permanente (steady state), em que as grandezas envolvidas no processo não sofrem alteração no tempo. Dessa forma, pode-se dizer que para efeito de projeto ou planejamento da operação, para períodos considerados longos, geralmente maiores que duas a três vezes a idade do lodo, a hipótese do regime permanente pode ser aplicada com segurança.

A taxa de aplicação de sólidos (TAS) é baseada na vazão afluente ao sistema biológico (Q), bem como a vazão oriunda da recirculação $\left(Q_{R}\right)$, pois o importante é que a carga aplicada por unidade de área não ultrapasse o fluxo limite de sólidos. Sendo assim, a carga afluente é a carga efetivamente aplicada ao decantador secundário, ou seja, $\left(Q_{+}+Q_{R}\right) \cdot X$.

A Tabela 1 apresenta, resumidamente, alguns parâmetros básicos largamente utilizados em projetos de sistemas de lodo ativados, baseados na experiência dos autores e de dados disponíveis em várias ETEs.
Tabela 1 - Principais características dos sistemas de lodos ativados

\begin{tabular}{|c|c|c|}
\hline \multirow[b]{2}{*}{ Parâmetro/Valores Típicos } & \multicolumn{2}{|c|}{ Sistema de Lodos Ativados } \\
\hline & Convencional & $\begin{array}{l}\text { Aeração } \\
\text { Prolongada }\end{array}$ \\
\hline Tempo de Detenção (h) & $6-8$ & $16-24$ \\
\hline Idade do Lodo (dias) & $4-10$ & $18-30$ \\
\hline SSVTA (mg/L) & $1.500-3.000$ & $3.000-6.000$ \\
\hline $\mathrm{A} / \mathrm{M}$ (kgDBO/kgSSV.d) & $0,3-0,8$ & $0,08-0,15$ \\
\hline Taxa de reciclo de lodo (\%) & 25 a 75 & 75 a 150 \\
\hline Remoção DQOO (\%) & $85-90$ & $90-95$ \\
\hline $\begin{array}{l}\text { SST no lodo de retorno (mg/L) } \\
\text { fundo do DS }\end{array}$ & $8.000-12.000$ & $8.000-12.000$ \\
\hline $\begin{array}{l}\text { Taxa de Aplicação hidráulica } \\
\left(\mathrm{m}^{3} / \mathrm{m}^{2} . \mathrm{h}\right) \text { para Q méd }\end{array}$ & $0,67-1,33$ & $0,33-0,67$ \\
\hline $\begin{array}{c}\text { Taxa de Aplicação hidráulica } \\
\left(\mathrm{m}^{3} / \mathrm{m}^{2} . \mathrm{h}\right) \text { para Q máx }\end{array}$ & $1,7-2,00$ & $1,00-1,33$ \\
\hline $\begin{array}{l}\text { Taxa de Aplicação de sólidos } \\
\left(\mathrm{kg} / \mathrm{m}^{2} . \mathrm{h}\right) \text { para Q med }\end{array}$ & $4,0-6,0$ & $1,0-5,0$ \\
\hline $\begin{array}{l}\text { Taxa de Aplicação hidráulica } \\
\left(\mathrm{kg} / \mathrm{m}^{2} . \mathrm{h}\right) \text { para Q máx }\end{array}$ & 10 & 7 \\
\hline
\end{tabular}

Adaptado de Alem Sobrinho e Kato (1.999) e von Sperling et al. (2.002).

Já a Taxa de Consumo de Oxigênio (TCO) é o parâmetro fundamental na determinação da potência do sistema de sopradores e, consequentemente, tem sua importância aumentada, já que está intimamente ligada ao consumo de energia elétrica da planta.

Segundo van Haandel (1999), 1 kg de DQO é oxidado por $1 \mathrm{~kg}$ de oxigênio $\left(\mathrm{O}_{2}\right)$, portanto o fluxo material orgânico oxidado será sempre numericamente igual ao fluxo do oxigênio consumido no processo de oxidação. O fluxo de oxigênio consumido é dado pelo produto do volume do reator pela Taxa de Consumo de Oxigênio. A TCO é igual ao consumo do oxigênio por unidade de volume e de tempo, e pode ser mensurada experimentalmente.

Assim, ao se fixar a idade do lodo desejada à operação e adotar a DQO na entrada do sistema biológico, bastaria, uma vez obedecido o equacionamento exposto a seguir, operar a planta dentro de limites pré-

\footnotetext{
${ }^{1}$ Segundo van Haandel e Marais (1999), parte do material orgânico afluente biodegradável (que tem DBO) no sistema de lodo ativado se transforma em resíduo endógeno não biodegradável, e que, portanto não tem DBO. Desse modo, a DBO "desaparece" no sistema de lodo ativado, sem que haja oxidação correspondente. Ou seja, os fluxos da DBO no efluente e no lodo de excesso, somados ao fluxo do oxigênio consumido pela oxidação do material orgânico sempre serão menores que o fluxo da DBO efluente'.
} 
-estabelecidos para a Taxa de Aplicação de Sólidos e da concentração dos sólidos voláteis no reator.

\subsection{Equacionamento}

Para o trabalho em questão, recorreu-se à cinética de lodos ativados proposta por Ekama, van Haandel e Marais, e consolidada pelo ASM1 da IWA (2008).

Os modelos matemáticos mais empregados na predição de comportamentos biológicos em unidades de tratamento de esgotos derivam dos estudos realizados pelo grupo de pesquisadores da IWA - International Water Association, denominados de ASM - Activated Sludge Models (Modelos para Lodo Ativado). Para esses modelos contribuíram os estudos realizados pela equipe de pesquisadores da Universidade de Cape Town na África do Sul, coordenada pelo professor G. Marais, em meados dos anos 80. Apesar de esses modelos se basearem em processos que ocorrem na biomassa suspensa, muitos pesquisadores que trabalham com biomassa fixa os utilizam, conforme preconizado por SEZERINO (2006).

A partição da DQQO, proposta primeiramente por van Haandel e Marais (1999), facilitou em muito o entendimento da dinâmica dos lodos ativados. Os autores utilizam a DQO (Demanda Química de Oxigênio) como parâmetro representativo da matéria orgânica, haja visto que é possível relacionar estequiometricamente e de forma direta, a DQO com o material orgânico. De acordo com esses autores, o material orgânico em termos de DQQ pode ser biodegradável e não biodegradável. A Figura 1 apresenta um esquema das frações do material orgânico em um sistema de lodo ativado.

Figura 1 - Representação das frações do material orgânico

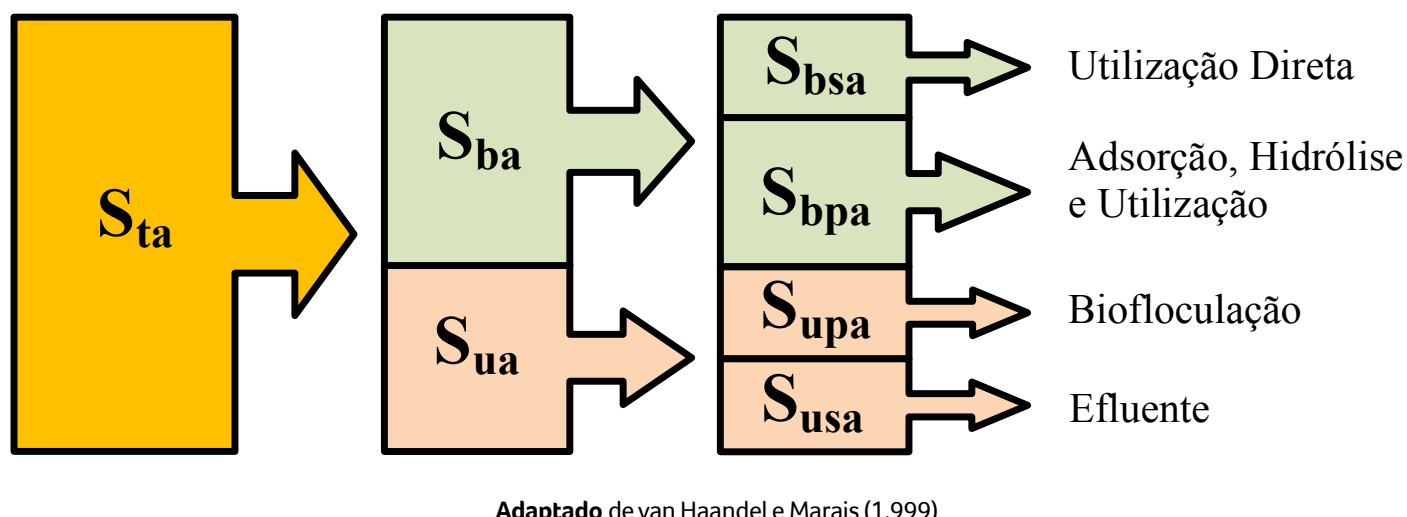

De acordo com o esquema da Figura 1, tem-se:

$\mathrm{S}_{\mathrm{ta}}-\mathrm{DQ} O$ afluente total

$\mathrm{S}_{\mathrm{ba}}$ - DQQO biodegradável afluente

$\mathrm{S}_{\text {ua }}$ - DQQO não biodegradável afluente

$\mathrm{S}_{\text {bsa }}$ - DQQO biodegradável solúvel afluente

$\mathrm{S}_{\mathrm{bpa}}$ - DQQO biodegradável particulada afluente

$\mathrm{S}_{\text {upa }}$ - DQQO não biodegradável particulada afluente

$\mathrm{S}_{\text {usa }}$ - DQQO não biodegradável solúvel afluente
E por meio dessa partição da DQ्O podem-se formular as frações:

$\mathrm{f}_{\text {us }}$ - fração da DQ̨O não biodegradável solúvel, que é o quociente entre a DQQ não biodegradável solúvel afluente e a DQQO total, ou seja, é a parte da DOQO que se perde pelo efluente final.

$\mathrm{f}_{\text {up }}$ - fração da DQQO não biodegradável particulada, que é o quociente entre a DQQO não biodegra- 
dável particulada afluente e a DQO total, ou seja,

$\begin{array}{ll}\mathrm{S}_{\mathrm{ua}}=\mathrm{S}_{\mathrm{ta}}-\mathrm{S}_{\mathrm{ba}} & {[5]} \\ \mathrm{S}_{\mathrm{upa}}=\mathrm{f}_{\mathrm{up}} \cdot \mathrm{S}_{\mathrm{ta}} & {[6]} \\ \mathrm{S}_{\mathrm{usa}}=\mathrm{f}_{\mathrm{us}} \cdot \mathrm{S}_{\mathrm{ta}} & {[7]} \\ \mathrm{S}_{\mathrm{ua}}=\left(\mathrm{f}_{\mathrm{us}}+\mathrm{f}_{\mathrm{up}}\right) \cdot \mathrm{S}_{\mathrm{ta}} & {[8]} \\ \mathrm{S}_{\mathrm{ba}}=\left(1-\mathrm{f}_{\mathrm{us}}-\mathrm{f}_{\mathrm{up}}\right) \cdot \mathrm{S}_{\mathrm{ta}} & {[9]}\end{array}$

A partir da partição da DQQO proposta, e em con$S_{\text {ba }}=\left(1-f_{\text {us }}-f_{\text {up }}\right) \cdot S_{\text {ta }}$ sonância com as frações definidas, fica fácil estabelecerem-se as seguintes relações:

$$
\begin{aligned}
& S_{\text {ta }}=S_{\text {ba }}+S_{\text {ua }} \\
& S_{\text {ba }}=\left(1-f_{\text {us }}-f_{\text {up }}\right) \cdot S_{\text {ta }} \\
& S_{\text {bsa }}=f_{\text {sb }} \cdot S_{\text {ba }} \\
& S_{\text {bpa }}=S_{\text {ba }}-S_{\text {bsa }}
\end{aligned}
$$

Da mesma forma que para a DQO foi proposta uma partição dos sólidos voláteis como se segue, na Figura 2:

Figura 2 - Esquema dos principais processos no sistema de lodos ativados.

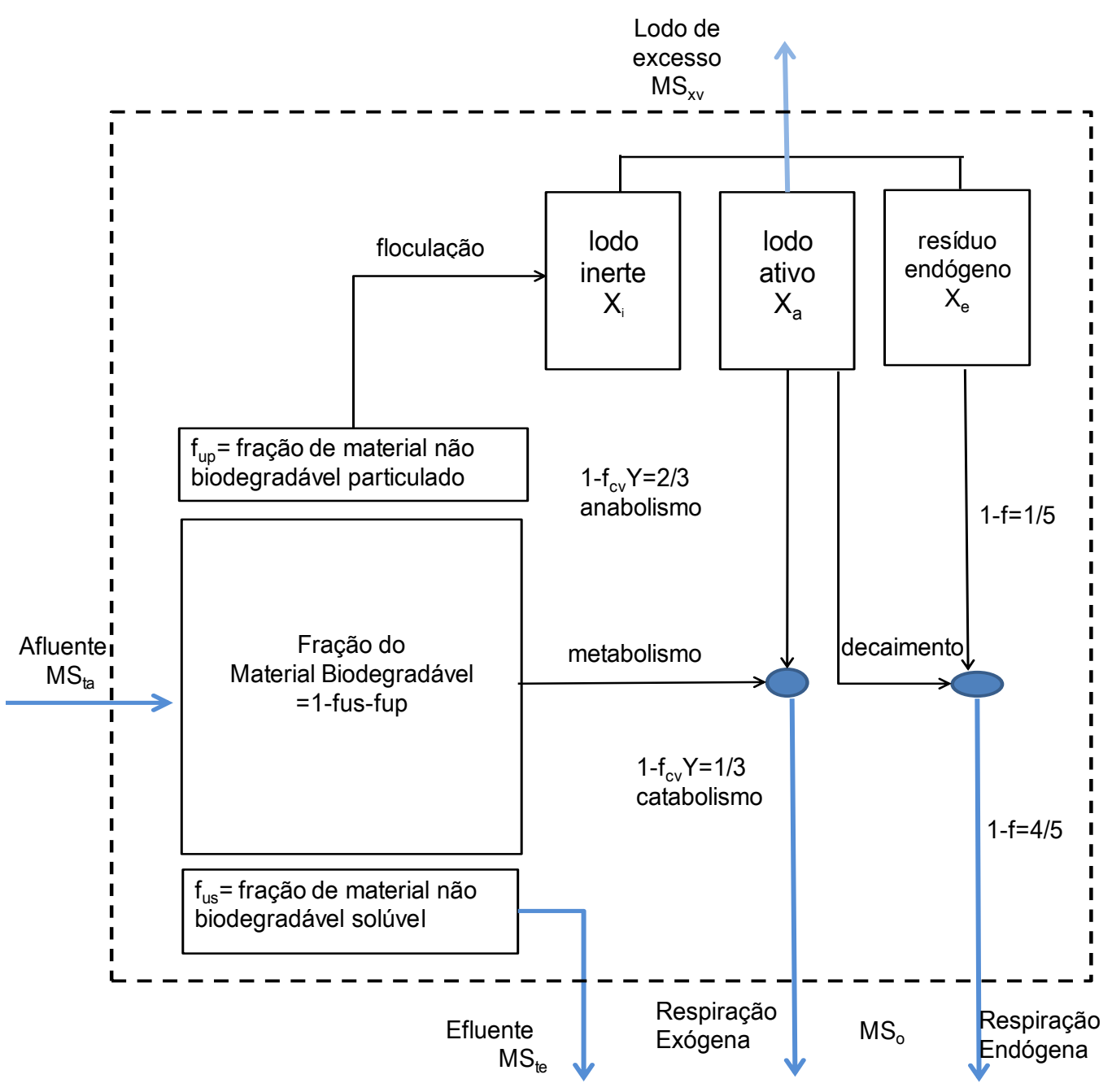

Adaptado de van Haandel e Marais (1.999) 
Acrescentando-se o conceito da idade do lodo, que é o tempo de permanência dos sólidos no sistema, definido como sendo a relação entre a massa do lodo presente no sistema e a massa descarregada por dia, ou seja:

$R_{s}=\frac{V_{r}}{q_{w}}$

Onde $V_{r}$ é o volume do reator e $\mathrm{q}_{\mathrm{w}}$ é a vazão de descarte do lodo.

Já o tempo de permanência do líquido no sistema, comumente chamado de tempo de detenção hidráulica, é dado pela razão entre o volume e a vazão afluente ao reator, ou seja:

$$
R_{h}=\frac{V_{r}}{Q}
$$

Acrescentando-se agora a definição da constante de decaimento do lodo ativo, $b_{H T}$, bem como a massa do lodo ativo presente no sistema por unida de DQQO biodegradável aplicada por dia, $\mathrm{C}_{\mathrm{r}}$, obtém-se :

$\mathrm{b}_{\mathrm{HT}}=0,24 \cdot 1,04^{(\mathrm{T}-20)}[12]$

Onde T é a temperatura em Celsius do licor misto e

$$
C_{r}=\frac{Y \cdot R_{s}}{1+b_{H T} \cdot R_{s}}
$$

Onde Y é o coeficiente de síntese celular, ou coeficiente de rendimento geralmente adotado com sendo 0,45 mg SSV/mg DQOO.

\section{$O$ lodo inerte $\left(X_{i}\right)$}

O lodo inerte que é gerado pela floculação do material orgânico não biodegradável particulado afluente, pode ser obtido por meio do balanço:

$$
\mathrm{Q} \cdot \mathrm{X}_{\mathrm{ia}}=\mathrm{q} \cdot \mathrm{X}_{\mathrm{i}}
$$

Onde $\mathrm{X}_{\mathrm{ia}}$ é a concentração dos sólidos inertes afluente.

Relaciona-se assim, a concentração dos sólidos orgânicos não biodegradáveis particulados ao material orgânico afluente por meio do fator de conversão $f_{c v}$, constante dada pela relação entre a DQO e os SSV (sólidos suspensos voláteis) no reator (algo entre 1,48 e 1,50) como se segue:

$$
\begin{aligned}
& X_{i a}=\frac{S_{u p a}}{f_{c v}}=\frac{f_{u p}}{f_{c v}} \cdot S_{t a}[15] \\
& X_{i}=\frac{f_{u p}}{f_{c v}} \frac{R_{s}}{R_{h}} S_{t a}
\end{aligned}
$$

\section{$O$ lodo ativo $\left(X_{a}\right)$}

O lodo ativo é afetado por três fatores: pelo seu crescimento devido à síntese celular, pelo seu decaimento e pela descarga do lodo em excesso. No regime permanente, onde não há variação da concentração do lodo ativo com o tempo tem-se:

$\frac{d X_{a}}{d t}=\left(\frac{d X_{a}}{d t}\right)_{c}+\left(\frac{d X_{a}}{d t}\right)_{d}+\left(\frac{d X_{a}}{d t}\right)_{e}=r_{c}+r_{d}+r_{e}=0[17]$

Assumindo-se que a taxa de crescimento do lodo ativo é proporcional à taxa de utilização do material biodegradável e que todo material biodegradável é utilizado, tem-se que a taxa de utilização do material biodegradável $r_{u}$ é igual à sua taxa $a$ de alimentação. Daí vem que:

$V_{r} \frac{d S_{b a}}{d t}=V_{r} \cdot r_{u}=Q \cdot S_{b a}$

$\mathrm{Ou}$

$r_{u}=\frac{Q \cdot S_{b a}}{V_{r}}=\frac{S_{b a}}{R_{h}}$ 
E como a taxa de crescimento do lodo ativo é dada como sendo:

$\left(\frac{d X_{a}}{d t}\right)_{c}=Y \cdot r_{u}=Y \frac{S_{b a}}{R_{h}}$

Para a taxa de decaimento do lodo ativo, assume-se uma reação de primeira ordem, de tal sorte que:

$\left(\frac{d X_{a}}{d t}\right)_{d}=r_{d}=-b_{H T} \cdot X_{a}$

Onde $\mathrm{b}_{\mathrm{HT}}$ é a constante de decaimento do lodo ativo

$E$, finalmente, para a taxa de diminuição do lodo ativo devido ao descarte do lodo, basta aplicar a definição da idade do lodo, que é a razão entre massa de lodo ativo pela taxa de descarga do lodo ativo:

$R_{s}=\frac{V_{r} X_{a}}{V_{r} \cdot\left(-\frac{d X_{a}}{d t}\right)_{e}}$

Portanto:

$\left(\frac{d X_{a}}{d t}\right)_{e}=r_{e}=-\frac{X_{a}}{R_{s}}$

Lembrando que:

$r_{c}+r_{d}+r_{e}=0$

Fica fácil, agora, chegar-se à expressão para o lodo ativo:

$X_{a}=\left[\frac{\left(1-f_{u s} f_{u p}\right) \cdot Y \cdot R_{s}}{1+b_{H T} \cdot R_{s}}\right] \cdot \frac{S_{t a}}{R_{h}}=\frac{\left(1-f_{u s}-f_{u p}\right) \cdot C_{r} \cdot S_{t a}}{R_{h}}$

\section{0 resíduo endógeno}

Assumindo-se que a taxa de produção de resíduo endógeno é igual à taxa de descarte do lodo de excesso e que a taxa de diminuição da concentração de resíduo endógeno devido à descarga do lodo pode ser calculada de modo semelhante à utilizada no lodo ativo, vem que:

$X_{e}=f \cdot b_{H T} \cdot R_{s} \cdot X_{a}$

Onde f é a fração de lodo ativo decaído transformado em resíduo endógeno, que em geral assume valor de 0,20.

\section{O lodo orgânico $\left(X_{\downarrow}\right)$}

Levando-se em conta que: $\mathrm{X}_{\mathrm{v}}=\mathrm{X}_{\mathrm{a}}+\mathrm{X}_{\mathrm{e}}+\mathrm{X}_{\mathrm{i}}$ vem:

$X_{v}=\left[\left(1-f_{u s}-f_{u p}\right) \cdot C_{r} \cdot\left(1+f \cdot b_{H T} \cdot R_{s}\right)+f_{u p} \frac{R_{s}}{f_{c v}}\right] \cdot \frac{S_{\text {ta }}}{R_{h}}[27]$

A concentração dos sólidos totais no reator será dada por:

$X_{T A}=\frac{X_{v}}{f_{v}}$

Onde $f_{v}$ representa a relação entre SSV eSST no reator

MetCalf \& Eddy (2003) apresentam valores típicos e de projetos para alguns parâmetros, conforme apresentado na Tabela 2.

Tabela 2 - Valores típicos em processos de lodos ativados

\begin{tabular}{|c|c|c|c|}
\hline & Rs & SST & Rh \\
\hline Fluxo em Pistão & $3-15$ & $1.000-3.000$ & $4-8$ \\
\hline Mistura Completa & $2-15$ & $1.000-5.000$ & $3-5$ \\
\hline $\begin{array}{c}\text { Alimentação } \\
\text { Escalonada }\end{array}$ & $3-15$ & $1.500-3.500$ & $3-5$ \\
\hline Aeração Modificada & $0,2-0,5$ & $200-1.000$ & $1,5-3$ \\
\hline $\begin{array}{c}\text { Estabilização por } \\
\text { Contato }\end{array}$ & $5-15$ & $\begin{array}{c}(1.000-3.000)^{\mathrm{a}} \\
(4.000-9.000)^{\mathrm{b}}\end{array}$ & $\begin{array}{c}(0,5-1,0)^{\mathrm{a}} \\
(3-6)^{\mathrm{b}}\end{array}$ \\
\hline Aeração Prolongada & $20-30$ & $1.500-5.000$ & $18-36$ \\
\hline Aeração de Alta Taxa & $5-10$ & $2.000-3.000$ & $2-4$ \\
\hline Oxigênio Puro & $3-10$ & $3.000-8.000$ & $4-8$ \\
\hline
\end{tabular}

a Unidade de contato

b Unidade de estabilização de sólidos

Adaptado de Metcalf \& Eddy (2.003). 


\subsection{As frações da DQO:}

Para as frações da DQQO afluente, valem as seguintes expressões:

Chamando-se de $\mathrm{ME}_{\mathrm{v}}$ da massa diária de lodo de excesso produzida por dia (mg SSV/d) e de $M_{V}$ a massa de lodo

$M E_{V}=\frac{V_{r} \cdot X_{V}}{R_{s}}=\left[\left(1-f_{u s}-f_{u p}\right) \cdot\left(1+f \cdot b_{H T} \cdot R_{s}\right) \cdot \frac{C_{r}}{R_{s}}+\frac{f_{u p}}{f_{c v}}\right] \cdot M S_{\text {ta }}$ [29]

$M X_{V}=V_{r} \cdot X_{V}=\left[\left(1-f_{u s}-f_{u p}\right) \cdot\left(1+f \cdot b_{H T} \cdot R_{s}\right) \cdot C_{r}+\frac{f_{u p} \cdot R_{s}}{f_{c v}}\right] \cdot M S_{t a}[30]$

Lembrando que a relação entre a DOQO e os sólidos suspensos voláteis é representeada pela constante fcr vem que:

$m S_{x v}=f_{c v} \cdot \frac{M E_{v}}{M X_{v}}=f_{c v} \cdot\left(1-f_{u s}-f_{u p}\right) \cdot\left(1+f \cdot b_{H T} \cdot R_{s}\right) \cdot \frac{C_{r}}{R_{s}}+f_{u p} \quad[31]$

\section{A fração do lodo oxidada $m S_{0}$}

A taxa de consumo de oxigênio devido a respiração exógena diz que para cada grama de DQQO se formam $Y$ gramas de lodo ativo, cuja DQO é de $f_{c v}, Y$, restando portanto, $\left(1-f_{c v} Y\right) g$ de DQO para a oxidação. Para essa oxidação será necessária, por definição, $\left(1-f_{c v} Y\right) g$ de oxigênio; daí, o consumo de oxigênio para a respiração exógena será uma fração $\left(1-f_{c v} Y\right)$ da DQO metabolizada. Donde:

$\operatorname{TCO}_{e x}=\left(1-f_{c v}\right) \cdot r_{u}=\left(1-f_{c v}\right) \cdot \frac{S_{b a}}{R_{h}}$

Sabendo-se que a taxa de oxidação do lodo ativo é a diferença entre as taxas de decaimento do lodo ativo e da taxa do surgimento do resíduo endógeno vem que:

$r_{o}=\left(\frac{d X_{a}}{d t}\right)_{d}-\left(\frac{d X_{e}}{d t}\right)_{d}=b_{H T} X_{a}-f \cdot b_{H T} \cdot X_{a}=(1-f) \cdot b_{H T} \cdot X_{a}[33]$
Usando-se, novamente a constante $f_{c v}$, pode-se escrever que:

$\operatorname{TCO}_{\text {en }}=f_{c v} \cdot r_{o}=f_{c v} \cdot(1-f) \cdot b_{H T} \cdot X_{a}$

Lembrando que a Taxa de Consumo de Oxigênio para o material orgânico será a soma das taxas para as respirações exógena e endógena:

$\mathrm{TCO}=\mathrm{TCO}_{\mathrm{ex}}+\mathrm{TCO}_{\text {en }}=\left(1-f_{\mathrm{cv}}\right) \cdot \frac{\mathrm{S}_{\mathrm{ba}}}{\mathrm{R}_{\mathrm{h}}}+\mathrm{f}_{\mathrm{cv}} \cdot(1-f) \cdot \mathrm{b}_{\mathrm{HT}} \cdot \mathrm{X}_{\mathrm{a}}$

Podendo-se chegar à expressão:

TCO $=\left(1-f_{u s}-f_{u p}\right) \cdot\left(1-f_{c v} \cdot Y+f_{c v} \cdot(1-f) \cdot b_{H T} \cdot C_{r}\right) \frac{S_{t a}}{R_{h}} \quad[36]$

A fração da DQQ que será oxidada no sistema de lodos ativados será expressa por:

$m S_{o}=\frac{M O_{c}}{M S_{t a}}=\frac{V_{r} \cdot T C O}{Q \cdot S_{t a}}=\left(1-f_{u s}-f_{u p}\right) \cdot\left[\left(1-f_{c v} \cdot Y\right)+f_{c v} \cdot(1-f) \cdot b_{H T} \cdot C_{r}\right][37]$

E, finalmente a fração do material orgânico que permanece na fase líquida será dada por:

$\mathrm{mS}_{\mathrm{te}}=\mathrm{f}_{\mathrm{us}}$

Lembrando sempre que:

$\mathrm{mS}_{\mathrm{xv}}+\mathrm{mS}_{\mathrm{o}}+\mathrm{mS}_{\mathrm{te}}=1[39]$

Lembrando sempre que:

$\mathrm{q}_{\mathrm{w}}=\frac{\mathrm{mS} \mathrm{xv}_{\mathrm{xv}} \cdot \mathrm{Q} \cdot \mathrm{S}_{\mathrm{ta}}}{\mathrm{f}_{\mathrm{cv}} \cdot \mathrm{X}_{\mathrm{w}}}$

Caso o descarte se faça diretamente pelo reator, a concentração de sólidos do descarte será a mesma do reator:

$\mathrm{X}_{\mathrm{w}}=\mathrm{X}_{\mathrm{TA}}$ 
Já a vazão de descarte será:

$$
\mathrm{q}_{\mathrm{w}}=\frac{\mathrm{V}_{\mathrm{r}}}{\mathrm{R}_{\mathrm{s}}}
$$

A carga de sólidos, em termos da DQQ , do descarte será dada por:

$\mathrm{L}_{\mathrm{w}}=\mathrm{Q} \cdot \mathrm{S}_{\mathrm{ta}} \cdot \mathrm{mS}_{\mathrm{xv}}$

A carga de sólidos que deixa a unidade de desaguamento, em DQQO, será dada por:

$\mathrm{L}_{\text {torta }}=$ cap_sol $\cdot \mathrm{L}_{\mathrm{w}}[44]$

A vazão do desague será dada por:

$\mathrm{Q}_{\text {torta }}=\frac{\mathrm{L}_{\text {torta }}}{\mathrm{DQO}_{\text {torta }}}[45]$

A vazão que deixa a ETE será dada por:

$Q_{\text {ef }}=Q-Q_{\text {torta }}$
E a vazão do reciclo, ou a vazão que retorna ao início do processo, será dada por:

$Q_{\text {rec }}=q_{w}-Q_{\text {torta }}$

Já a carga de sólidos que retorna ao início do processo por meio do reciclo será dada por:

$\mathrm{L}_{\text {rec }}=\mathrm{L}_{\mathrm{w}}-\mathrm{L}_{\text {torta }}$

Em termos de DQO, a carga que será somada à carga que aflui ao sistema biológico será:

Finalmente, para a taxa de aplicação de sólidos, recorre-se à expressão:

$\mathrm{TAS}=\frac{(\mathrm{Q}+\mathrm{RQ}) \cdot \mathrm{X}_{\mathrm{TA}}}{\text { Area }_{\mathrm{DS}}}$

Dessa forma, por meio das equações obtidas pode-se construir um modelo baseado em cargas (ou massa) de DQO de um sistema qualquer de lodos ativados, conforme o roteiro de cálculo que segue:

\begin{tabular}{|c|c|c|}
\hline \multicolumn{3}{|l|}{ Dados de Entrada } \\
\hline Parâmetros Físicos & Símbolos & Unidade \\
\hline Número de Decantadores s Secundários & $\mathrm{nDS}$ & \\
\hline Número de Tanques de Aeração & nTA & \\
\hline Diâmetro dos Decotadores Secundários & $\mathrm{D}$ & $\mathrm{m}$ \\
\hline Volume Unitário do Reator & $\mathrm{Vr}$ & $\mathrm{m}^{3}$ \\
\hline \multicolumn{3}{|l|}{ Parâmetros Operacionais } \\
\hline Vazão afluente à ETE & Q & $\mathrm{m} 3 / \mathrm{d}$ \\
\hline DQQO afluente à ETE & DQOa & $\mathrm{mg} / \mathrm{L}$ \\
\hline \multicolumn{3}{|l|}{ Fase Líquida } \\
\hline Idade do Lodo & Rs & $d$ \\
\hline Taxa de recirculação & $\mathrm{R}$ & $\%$ \\
\hline Relação SSV/SST nos reatores & fiv & \\
\hline \multicolumn{3}{|l|}{ Caso o descarte seja feito pelos Dec. Sec. } \\
\hline Concentração lodo de excesso & $X_{w}$ & $\mathrm{mg} / \mathrm{L}$ \\
\hline \multicolumn{3}{|l|}{ Fase Sólida } \\
\hline Captura de sólidos no desaguamento & Cap_Sol & $\%$ \\
\hline DQQO da torta & DQQOtorta & $\mathrm{mg} / \mathrm{L}$ \\
\hline Porcentagem de Sólidos Totais na torta & ST & $\%$ \\
\hline
\end{tabular}




\begin{tabular}{|c|c|c|c|}
\hline \multicolumn{4}{|l|}{ Dados de Entrada } \\
\hline Parâmetros cinéticos & Símbolos & Unidade & Val. típico \\
\hline Fração do lodo ativo que se torna resíduo endógeno & $\mathrm{f}$ & & 0,20 \\
\hline Fração da DQQ afluente ao reator não biodegr. sol. & fus & & 0,08 \\
\hline Fração da DQO afluente ao reator não biodegr. part & fup & & 0,12 \\
\hline Fração biodegradável solúvel afluente & fsb & & 0,20 \\
\hline Razão DQQO/SSV no lodo orgânico & fcr & $\mathrm{mgDQO} / \mathrm{mgSSV}$ & 1,48 \\
\hline Coeficiente de rendimento & Y & $\mathrm{mgSSV} / \mathrm{mgDQO}$ & 0,45 \\
\hline Temperatura & $\mathrm{T}$ & oC & \\
\hline Cálculos - Fase Líquida & Símbolos & Unidade & Equação \\
\hline Constante de decaimento do lodo & $\mathrm{b}_{\mathrm{HT}}$ & & [12] \\
\hline Lodo Ativo por unidade de massa de DQO aplicada por dia & $\mathrm{Cr}$ & & [13] \\
\hline Tempo de Detenção Hidráulica do Sistema & $\mathrm{Rh}$ & d & [11] \\
\hline \multicolumn{4}{|l|}{ DQO } \\
\hline DQO total afluente ao Reator & Sta & $\mathrm{mg} / \mathrm{L}$ & [dado] \\
\hline DQO biodegradável afluente & Sba & $\mathrm{mg} / \mathrm{L}$ & [2] \\
\hline DQQ biodegradável solúvel afluente (mg/L) & Sbsa & $\mathrm{mg} / \mathrm{L}$ & [3] \\
\hline DQO não biodegradável afluente & Sua & $\mathrm{mg} / \mathrm{L}$ & [5] \\
\hline DQO biodegradável particulada afluente (mg/L) & Sbpa & $\mathrm{mg} / \mathrm{L}$ & [4] \\
\hline DQO biodegradável solúvel da afluente (mg/L) & Susa & $\mathrm{mg} / \mathrm{L}$ & [7] \\
\hline DQO biodegradável particulada afluente (mg/L) & Supa & $\mathrm{mg} / \mathrm{L}$ & [6] \\
\hline Fração da DQQ afluente oxidada & mSo & & [37] \\
\hline Fração da DQO afluente no lodo de excesso & $\mathrm{mSxv}$ & & [31] \\
\hline Fração da DQO que permanece no sistema & mSte & & [38] \\
\hline Taxa de consumo de Oxigênio & TCO & mg/(L.h) & [36] \\
\hline \multicolumn{4}{|l|}{ Lodos } \\
\hline Lodo inerte (mg/L) & $\mathrm{Xi}$ & $\mathrm{mg} / \mathrm{L}$ & [16] \\
\hline Lodo Ativo (mg/L) & $\mathrm{Xa}$ & $\mathrm{mg} / \mathrm{L}$ & [25] \\
\hline Resíduo Endógeno (mg/L) & $\mathrm{Xe}$ & $\mathrm{mg} / \mathrm{L}$ & [26] \\
\hline Lodo volátil (mg/L) & $\mathrm{Xv}$ & $\mathrm{mg} / \mathrm{L}$ & [27] \\
\hline Concentração de sólidos totais no reator & Xta & $\mathrm{mg} / \mathrm{L}$ & [28] \\
\hline \multicolumn{4}{|l|}{ Cálculos - Fase Sólida } \\
\hline \multicolumn{4}{|c|}{ Caso o descarte seja efetuado por meio dos Decantadores Secundários } \\
\hline Vazão de descarte de lodo & qw & $\mathrm{m}^{3} / \mathrm{d}$ & [40] \\
\hline \multicolumn{4}{|l|}{ Caso o descarte se faça diretamente por meio do Reator } \\
\hline Concentração de descarte & $X_{w}=X_{t a}$ & $\mathrm{mg} / \mathrm{L}$ & [28] \\
\hline Vazão de descarte & qw & $\mathrm{m}^{3} / \mathrm{d}$ & [42] \\
\hline Taxa de aplicação de sólidos & TAS & $\mathrm{kg} /(\mathrm{m} 2 . \mathrm{h})$ & [49] \\
\hline Carga de sólidos totais no descarte & Lw & $\mathrm{kg} / \mathrm{d}$ & [48] \\
\hline Carga de sólidos na torta & Ltorta & $\mathrm{kg} / \mathrm{d}$ & [44] \\
\hline Vazão de torta gerada & Qtorta & $\mathrm{m} 3 / \mathrm{d}$ & [45] \\
\hline Vazão do efluente final & Qef & $\mathrm{m} 3 / \mathrm{d}$ & [46] \\
\hline Vazão de recirculação & Qrec & $\mathrm{m} 3 / \mathrm{d}$ & [47] \\
\hline Carga de DQO que retorna ao início do processo & Lrec & $\mathrm{kg} / \mathrm{d}$ & [48] \\
\hline
\end{tabular}

\section{Iterações}

\begin{abstract}
A vazão afluente ao sistema biológico é somada à vazão do reciclo, bem como as cargas de SST e de DQO até que haja a convergência, que ocorre rapidamente, geralmente entre a terceira e a quarta iteração.
\end{abstract}

\subsection{Estudo de caso - Bragança Paulista}

O município de Bragança Paulista está localizado na região nordeste do Estado de São Paulo, limitando-se com os municípios de Atibaia, Itatiba, Morungaba, Tuiuti, Pedra Bela, Pinhalzinho, Vargem e Piracaia. Possui uma área total de 513,56 
$\mathrm{km}^{2}$, e o principal acesso ao município, a partir de São Paulo, é feito pela Rodovia Fernão Dias.

A cidade iniciou, no ano de 2010, a construção da ETE - Estação de Tratamento de Esgoto. Esse projeto, na fase de implantação, enfrentou problemas relacionados à execução das obras, o que resultou na necessidade de mudança locacional e de layout.
Além disso, por solicitação do órgão ambiental estadual, Cetesb, adequações foram realizadas visando adaptar a ETE projetada à realidade da cidade, bem como às demandas futuras. Esquematicamente, a ETE pode ser representada como no fluxograma da Figura 3.

Figura 3 - Esquema da ETE Bragança Paulista

\section{Fluxograma de processo - ETE Bragança}

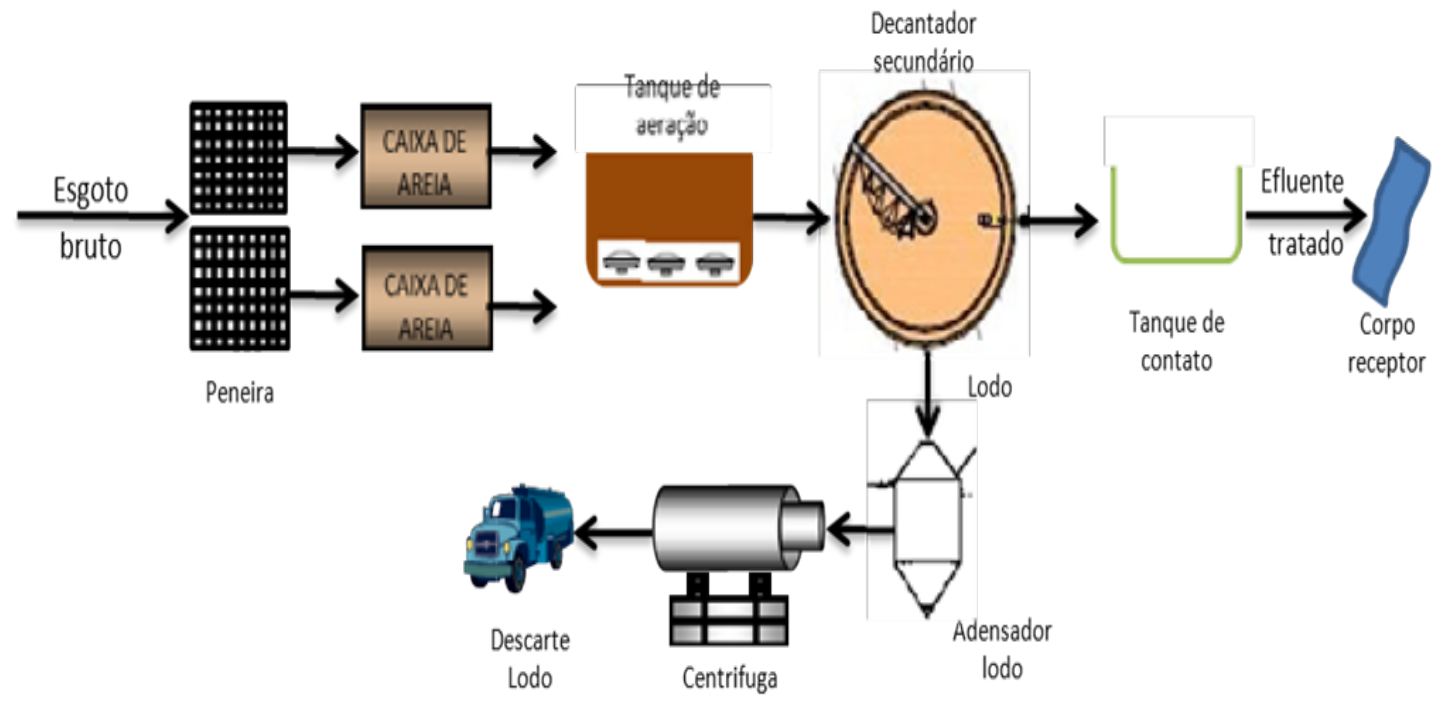

Adaptado de Sistemas Isolados - Sabesp [2.011].

Assim, para a situação atual da ETE Bragança Paulista (2011), considerando-se uma vazão média de $100 \mathrm{~L} / \mathrm{s}$ e uma concentração de DQQO de $500 \mathrm{mg} / \mathrm{L}$, com descarte de lodo pelo decantador secundário a uma concentração de $8.000 \mathrm{mg} / \mathrm{L}$, (haja visto que a planta não possui decantadores primários), a melhor configuração operacional procurada seria aquela em que o número de unidades de operação fosse o mínimo possível. Espera-se, também, que a planta opere com a menor taxa de consumo de oxigênio, para uma mesma idade do lodo, respeitando-se os limites para as taxas de aplicação de sólidos.
Para a fase inicial de operação $(Q=100 \mathrm{~L} / \mathrm{s}) \mathrm{z}$ adotaram-se os seguintes valores:

Com esses dados obtiveram-se como saída do modelo os seguintes resultados, conforme mostram as Tabelas 3 e 4:

\begin{tabular}{|l|c|}
\hline Valores adotados & \\
\hline Dados Operacionais & $\mathrm{Q}=8.640$ \\
\hline Vazão Afluente $\left(\mathrm{m}^{3} / \mathrm{d}\right)$ & $\mathrm{S}_{\mathrm{ta}}=500$ \\
\hline $\begin{array}{l}\text { Demanda Química de Oxigênio afluente aos } \\
\text { reatores }(\mathrm{mg} / \mathrm{L})\end{array}$ & $\mathrm{V}=9.408$ \\
\hline Volume de cada reator $\left(\mathrm{m}^{3}\right)$ & $\mathrm{D}=24$ \\
\hline Diâmetro de cada decantador secundário $(\mathrm{m})$ & $\mathrm{nTA}(1 \mathrm{a} 4)$ \\
\hline Número de tanques de aeração & $\mathrm{nDS}(1 \mathrm{a} 4)$ \\
\hline Número de decantadores secundários & \\
\hline
\end{tabular}




\begin{tabular}{|c|c|}
\hline Parâmetros Operacionais Admitidos & \\
\hline Taxa de recirculação de lodo (\%) & $Q_{R} / Q=1$ \\
\hline $\begin{array}{l}\text { Concentração de SST no descarte de lodo (mg/L) } \\
\text { (pelo DS) }\end{array}$ & $x_{w}=8.000$ \\
\hline Temperatura média do licor misto $\left({ }^{\circ} \mathrm{C}\right)$ & $T=22$ \\
\hline \multicolumn{2}{|l|}{ Parâmetros Cinéticos } \\
\hline $\begin{array}{l}\text { Fração de DQO não biodegradável solúvel } \\
\text { afluente }\end{array}$ & $\mathrm{f}_{\mathrm{us}}=0,08$ \\
\hline $\begin{array}{l}\text { Fração de DQO não biodegradável particulada } \\
\text { afluente }\end{array}$ & $f_{u p}=0,12$ \\
\hline Fração do lodo que se torna resíduo endógeno & $f=0,20$ \\
\hline Fração biodegradável solúvel afluente & $\mathrm{f}_{\mathrm{sb}}=0,25$ \\
\hline Razão da DQQ/SSV no lodo orgânico & $f_{c v}=1,48$ \\
\hline Razão SSV/SST no licor misto & $\mathrm{f}_{\mathrm{v}}=0,75$ \\
\hline Coeficiente de síntese celular (mgSSV/mgDQO) & $Y=0,45$ \\
\hline \multicolumn{2}{|l|}{ Parâmetros a serem atingidos } \\
\hline Idade do Lodo (d) & $R_{s}=29,5$ \\
\hline Taxa de Consumo de Oxigênio (mg/(L.h)) & TCO \\
\hline Taxa de Aplicação de Sólidos (kg/(m².h)) & $\operatorname{TAS}(2$ a 7$)$ \\
\hline \multicolumn{2}{|l|}{ Fase Sólida } \\
\hline Captura de sólidos no desaguamento & Cap_Sol $95 \%$ \\
\hline DQO da torta (mg DQO/L) & 300.000 \\
\hline Concentração de ST na torta & 250.000 ou $25 \%$ \\
\hline
\end{tabular}

Tabela 3 - Dados de Entrada e Saída da ETE

\begin{tabular}{|c|c|c|}
\hline \multicolumn{3}{|c|}{ AFLUENTE A ETE } \\
\hline Q & $\mathrm{m}^{3} / \mathrm{d}$ & 8.640 \\
\hline DQO & $\mathrm{mg} / \mathrm{L}$ & 500 \\
\hline \multicolumn{3}{|c|}{ REATOR + DS } \\
\hline MSta & $\mathrm{kg} / \mathrm{d}$ & 4.320 \\
\hline MSo & $\mathrm{kg} / \mathrm{d}$ & 4.320 \\
\hline MSxv & $\mathrm{kg} / \mathrm{d}$ & 2.783 \\
\hline MSte & $\mathrm{kg} / \mathrm{d}$ & 1.191 \\
\hline LDQOrec & $\mathrm{kg} / \mathrm{d}$ & 346 \\
\hline mSo & - & 60 \\
\hline mSxv & - & 0,64 \\
\hline mSte & - & 0,28 \\
\hline
\end{tabular}

Tabela 4 - Resultados das Iterações.

\begin{tabular}{|c|c|c|c|c|c|c|c|c|c|c|c|c|}
\hline & & $i=1$ & $i=2$ & $i=3$ & $\mathrm{i}=4$ & $i=5$ & $i=6$ & $\mathrm{i}=7$ & $i=8$ & $\mathrm{i}=9$ & $i=10$ & $i=11$ \\
\hline \multicolumn{13}{|c|}{ AFLUENTE A ETE } \\
\hline Q & $\mathrm{m}^{3} / \mathrm{d}$ & 8.640 & 8.793 & 8.796 & 8.796 & 8.796 & 8.796 & 8.796 & 8.796 & 8.796 & 8.796 & 8.796 \\
\hline Sta & $\mathrm{mg} / \mathrm{L}$ & 500 & 500 & 502 & 502 & 502 & 502 & 502 & 502 & 502 & 502 & 502 \\
\hline MSta & kg DQ:O/d & 4.320 & 4.414 & 4.416 & 4.416 & 4.416 & 4.416 & 4.416 & 4.416 & 4.416 & 4.416 & 4.416 \\
\hline Susa & $\mathrm{mg} / \mathrm{L}$ & 40 & 40 & 40 & 40 & 40 & 40 & 40 & 40 & 40 & 40 & 40 \\
\hline Sba & $\mathrm{mg} / \mathrm{L}$ & 400 & 400 & 402 & 402 & 402 & 402 & 402 & 402 & 402 & 402 & 402 \\
\hline Supa & $\mathrm{mg} / \mathrm{L}$ & 60 & 60 & 60 & 60 & 60 & 60 & 60 & 60 & 60 & 60 & 60 \\
\hline Sua & $\mathrm{mg} / \mathrm{L}$ & 100 & 100 & 100 & 100 & 100 & 100 & 100 & 100 & 100 & 100 & 100 \\
\hline Sbsa & $\mathrm{mg} / \mathrm{L}$ & 80 & 80 & 80 & 80 & 80 & 80 & 80 & 80 & 80 & 80 & 80 \\
\hline Sbpa & $\mathrm{mg} / \mathrm{L}$ & 320 & 32 & 32 & 32 & 32 & 32 & 32 & 32 & 32 & 32 & 32 \\
\hline $\mathrm{Rh}$ & (d) & 1,09 & 1,07 & 1,07 & 1,07 & 1,07 & 1,07 & 1,07 & 1,07 & 1,07 & 1,07 & 1,07 \\
\hline$X i$ & $\mathrm{mg} / \mathrm{L}$ & 149 & 152 & 152 & 152 & 152 & 152 & 152 & 152 & 152 & 152 & 152 \\
\hline $\mathrm{Xa}$ & $\mathrm{mg} / \mathrm{L}$ & 324 & 330 & 332 & 332 & 332 & 332 & 332 & 332 & 332 & 332 & 332 \\
\hline $\mathrm{Xe}$ & $\mathrm{mg} / \mathrm{L}$ & 67 & 69 & 69 & 69 & 69 & 69 & 69 & 69 & 69 & 69 & 69 \\
\hline$X v$ & $\mathrm{mg} / \mathrm{L}$ & 541 & 550 & 553 & 553 & 553 & 553 & 553 & 553 & 553 & 553 & 553 \\
\hline \multicolumn{13}{|c|}{ REATOR BIOLÓGICO } \\
\hline Xta & $\mathrm{mg} / \mathrm{L}$ & 721 & 734 & 737 & 737 & 737 & 737 & 737 & 737 & 737 & 737 & 737 \\
\hline TCO & mg/L.h & 9,27 & 9,43 & 9,47 & 9,47 & 9,47 & 9,47 & 9,47 & 9,47 & 9,47 & 9,47 & 9,47 \\
\hline \multicolumn{13}{|c|}{ DESCARTE PELO DECANTADOR SECUNDÁRIO } \\
\hline$X_{w}$ & mg SSV/L & 8.000 & 8.000 & 8.000 & 8.000 & 8.000 & 8.000 & 8.000 & 8.000 & 8.000 & 8.000 & 8.000 \\
\hline$X w$ & $\mathrm{mg} \mathrm{DQO} / \mathrm{L}$ & 11.840 & 11.840 & 11.840 & 11.840 & 11.840 & 11.840 & 11.840 & 11.840 & 11.840 & 11.840 & 11.840 \\
\hline Qw & $\mathrm{m}^{3} / \mathrm{d}$ & 159 & 162 & 162 & 163 & 163 & 163 & 163 & 163 & 163 & 163 & 163 \\
\hline MSxv & $\mathrm{kg} \mathrm{DQQO/d}$ & 1.882 & 1.923 & 1.924 & 1.924 & 1.924 & 1.924 & 1.924 & 1.924 & 1.924 & 1.924 & 1.924 \\
\hline Lw & $\mathrm{kg} \mathrm{DQQO/d}$ & 1.882 & 1.923 & 1.924 & 1.924 & 1.924 & 1.924 & 1.924 & 1.924 & 1.924 & 1.924 & 1.924 \\
\hline Ltorta & $\mathrm{kg} \mathrm{DQQO/d}$ & 1.788 & 1.827 & 1.828 & 1.828 & 1.828 & 1.828 & 1.828 & 1.828 & 1.828 & 1.828 & 1.828 \\
\hline Lrec & kg DQQO/d & 94 & 96 & 96 & 96 & 96 & 96 & 96 & 96 & 96,20 & 96,20 & 96,20 \\
\hline Ltorta & $\mathrm{kg} \mathrm{DQQO/d}$ & 1.788 & 1.827 & 1.828 & 1.828 & 1.828 & 1.828 & 1.828 & 1.828 & 1.828 & 1.828 & 1.828 \\
\hline Q rec & $\mathrm{m}^{3} / \mathrm{d}$ & 153 & 156 & 156 & 156 & 156 & 156 & 156 & 156 & 156 & 156 & 156 \\
\hline Q torta & $\mathrm{m}^{3} / \mathrm{d}$ & 5,96 & 6,09 & 6,09 & 6,09 & 6,09 & 6,09 & 6,09 & 6,09 & 6,09 & 6,09 & 6,09 \\
\hline Qef & $\mathrm{m}^{3} / \mathrm{d}$ & 8.634 & 8.787 & 8.790 & 8.790 & 8.790 & 8.790 & 8.790 & 8.790 & 8.790 & 8.790 & 8.790 \\
\hline TAS & $\mathrm{kg} / \mathrm{m} 2 . \mathrm{d}$ & 1,15 & 1,19 & 1,19 & 1,19 & 1,19 & 1,19 & 1,19 & 1,19 & 1,19 & 1,19 & 1,19 \\
\hline
\end{tabular}


Tabela 5 - Resultados das Simulações.

\begin{tabular}{|c|c|c|c|c|c|c|c|c|c|c|}
\hline \multicolumn{2}{|c|}{ Rs $=29,5$ dias } & \multicolumn{9}{|c|}{ Assumindo $\mathrm{ST}=25 \%$ e $\rho=1,2 \mathrm{t} / \mathrm{m}^{3}$} \\
\hline Q & Xta & TAS & Dec.Sec & Reatores & TCO & mSo & $\mathrm{mSxv}$ & Q torta & $\begin{array}{c}\text { Massa } \\
\text { da torta }\end{array}$ & $\begin{array}{c}\text { Masa } \\
\text { seca }\end{array}$ \\
\hline$(\mathrm{L} / \mathrm{s})$ & $(\mathrm{mg} / \mathrm{L})$ & $\mathrm{kg} / \mathrm{m}^{2} . \mathrm{d}$ & \multicolumn{2}{|c|}{ em operação } & mg/L.h & $\%$ & $\%$ & $\mathrm{~m}^{3} / \mathrm{d}$ & $t / d$ & $t / d$ \\
\hline 100 & 3.412 & 5,5 & 1 & 1 & 18,95 & \multirow{4}{*}{64} & \multirow{4}{*}{28} & \multirow{2}{*}{3,83} & \multirow{2}{*}{4,60} & \multirow{2}{*}{1,15} \\
\hline 100 & 1.706 & 2,7 & 1 & 2 & 9,47 & & & & & \\
\hline 200 & 6.824 & 22 & 1 & 1 & 18,95 & & & \multirow{2}{*}{7,65} & \multirow{2}{*}{9,18} & \multirow{2}{*}{2,30} \\
\hline 200 & 3.413 & 5,5 & 2 & 2 & 9,47 & & & & & \\
\hline \multicolumn{2}{|c|}{ Rs $=4$ dias } & \multicolumn{9}{|c|}{ Assumindo $\mathrm{ST}=25 \%$ e $\rho=1,2 \mathrm{t} / \mathrm{m}^{3}$} \\
\hline $\mathbf{Q}$ & Xta & TAS & Dec.Sec & Reatores & TCO & mSo & $\mathrm{mSxv}$ & Q torta & $\begin{array}{c}\text { Massa } \\
\text { da torta }\end{array}$ & $\begin{array}{c}\text { Masa } \\
\text { seca }\end{array}$ \\
\hline$(\mathrm{L} / \mathrm{s})$ & (mg/L) & $\mathrm{kg} / \mathrm{m} 2 . \mathrm{d}$ & \multicolumn{2}{|c|}{ em operação } & mg/L.h & $\%$ & $\%$ & $\mathrm{~m} 3 / \mathrm{d}$ & $t / d$ & $\mathrm{t} / \mathrm{d}$ \\
\hline 100 & 737 & 1,2 & 1 & 1 & 9,47 & \multirow{3}{*}{48} & \multirow{3}{*}{44} & 6,09 & 7,31 & 1,83 \\
\hline 200 & 1.474 & 4,8 & 1 & 1 & 18,95 & & & \multirow{2}{*}{12,19} & \multirow{2}{*}{14,63} & \multirow{2}{*}{3,66} \\
\hline 200 & 737 & 1,2 & 2 & 2 & 9,47 & & & & & \\
\hline
\end{tabular}

\section{CONCLUSÕES}

Pelos resultados obtidos, para a vazão de $100 \mathrm{~L} / \mathrm{s}$, fica claro que a ETE é capaz de operar com um tanque de aeração e um decantador secundário, pois consegue manter a taxa de aplicação de sólidos dentro de valores aceitáveis. Entretanto, se quisermos operar com um pouco mais de folga, como a equipe operacional sugeriu, basta entrar com mais um tanque de aeração para diminuir a concentração de sólidos no reator, que cairia de 3.300 mg/L para níveis mais compatíveis com a experiência operacional, algo em torno de $1.700 \mathrm{mg} / \mathrm{L}$.

Já em um segundo cenário, ao se dobrar a vazão, porém mantendo-se os demais parâmetros inalteráveis, verifica-se que as taxas de aplicação de sólidos não conseguem operar em níveis razoáveis, havendo perda de sólidos pelo efluente, o que torna imprescindível a entrada de pelo menos mais um módulo da planta, ou seja, mais um decantador e um reator. Dessa forma, a taxa de aplicação de sólidos cairia de $22 \mathrm{~kg} / \mathrm{m}^{2}$.h para valores bem mais aceitáveis, da ordem de $5,5 \mathrm{~kg} / \mathrm{m}^{2} . h$.

Com relação ao consumo de energia elétrica, pelo fato de a ETE ter sido projetada para aeração prolongada (Rs=29,5 dias), verifica-se que haverá um consumo considerável de energia elétrica, já que $64 \%$ do total da DQO afluente deverá consumir energia necessária para oxidá-la. Entretanto, o custo com transporte de lodo seria reduzido para algo em torno de $28 \%$.

Em vez de operar a ETE com aeração prolongada, há a opção de reduzir a idade do lodo para valores convencionais (entre 4 e 10 dias). Assim, verifica-se uma redução significativa do consumo de energia elétrica (algo em torno de $48 \%$ da DQO total afluente), acarretando, no entanto, um significativo aumento do custo de transporte (que subiria dos $28 \%$ para algo na faixa de $44 \%$ ). Pelo fato de não se operar com aeração prolongada, há necessidade da construção de unidades de estabilização química. Em contrapartida, há a vantagem de se operar com menos unidades no sistema biológico, ou seja, 1 decantador e 1 reator, para uma vazão de 200 L/s.

Como, na época do projeto, o condicionante principal era o custo de transporte, previu-se que a ETE deveria trabalhar com aeração prolongada. A Tabela 5 ilustra as conclusões do projeto.

\section{REFERÊNCIAS}

van HAANDEL A., MARAIS G. (1999) - O comportamento do Sistema de Lodo Ativado - Teoria e Aplicações para Projetos e Operação. 
von SPERLING M. (2002) - Lodos Ativados - Princípios do Tratamento Biológico de Águas Residuárias, vol 4, DESA (Departamento de Engenharia Sanitária e Ambiental).

METCALF \& EDDY. (2003). Inc. Wastewater engineering: treatment and reuse. $4^{\mathrm{a}}$ ed. New York: McGraw-Hill.

SABESP - Shs (2011) - Elaboração de projeto executivo de adequação e ampliação das unidades de processo da ETE do Município de Bragança Paulista (RT-2 - RPR - 2 Tomo I) e Sistemas Isolados da Unidade de Negócios de Tratamento de Esgoto da Metropolitana MT-2016.

ALEM SOBRINHO, P.; KATO, M.T (1999) Análise crítica do uso do processo anaeróbio pra tratamento de esgotos sanitários. Rio de Janeiro, ABES-RJ.

SEZERINO, P. H. (2006). Potencialidade dos filtros plantados com macrófitas (constructed wetlands) no pós-tratamento de lagoas de estabilização sob condições de clima subtropical. Florianópolis - SC. Programa de Pós Graduação em Engenharia Ambiental, UFSC.

HENZE M., VAN LOOSDRECHT M., EKAMA G., BRDJANOVIC D.- Biological Wastewater Treatment - Principles, Modelling and Design - IWA Publishing 2008, UK.
Region-wide Wastewater Treatment Plant Modeling Enhances Facility Management and Treatment - Principles. Modeling and Design - IWA Publishing, 2008 ,UK.

Region-wide Wastewater Treatment Plant Modeling Enhances Facility Management and Planning Christopher M. Bye , José R. Bicudo, Richard M. Jones, 2012, WEFTEC

GIORDANO, G. (2010) - Avaliação de efeito tóxico utilizando o respirômetro em processos de lodos ativados. Dissertação de mestrado, Pontifícia da Universidade Católica do Rio de Janeiro.

VICENTE V. R, (2006) - Modelos de operação para centros de controle de Sistemas de Abastecimento de Água - Estudo de Caso Sistema Adutor Metropolitano de São Paulo. Dissertação de mestrado, Universidade de São Paulo (USP).

ALEM SOBRINHO, P (2012) - Aspectos Técnicos Relevantes para - Dimensionamento de Sistemas de Tratamento Biológico Aeróbio e Anaeróbio (Escola Politécnica da USP).

FERNANDES A.N et al (2006) - Termodinâmica Química (Ed. Fernandes, Pizzo \& Moraes Jr)

HELOU, L. C. (2000) Tratamento de esgotos convencionais por lodos ativados com aproveitamento dos efluentes para reúso. Tese (Doutorado em Engenharia Hidráulica e Sanitária) - Universidade de São Paulo (USP). 